\title{
Cognitive Function and Health Literacy Decline in a Cohort of Aging English Adults
}

\author{
Lindsay C. Kobayashi, MSc' , Jane Wardle, $P h D^{7}$, Michael S. Wolf, $P h D^{2}$, and \\ Christian von Wagner, $\mathrm{PhD}^{\prime}$
}

${ }^{1}$ Health Behaviour Research Centre, Department of Epidemiology and Public Health, University College London, London, UK; ${ }^{2}$ Division of General Internal Medicine, Feinberg School of Medicine, Northwestern University, Chicago, IL, USA.

BACKGROUND: Low health literacy is common among aging patients and is a risk factor for morbidity and mortality. We aimed to describe health literacy decline during aging and to investigate the roles of cognitive function and decline in determining health literacy decline.

METHODS: Data were from 5,256 non-cognitively impaired adults aged $\geq 52$ years in the English Longitudinal Study of Ageing. Health literacy was assessed using a four-item reading comprehension assessment of a fictitious medicine label, and cognitive function was assessed in a battery administered in-person at baseline (20042005) and at follow-up (2010-2011).

RESULTS: Overall, $19.6 \%(1,032 / 5,256)$ of participants declined in health literacy score over the follow-up. Among adults aged $\geq 80$ years at baseline, this proportion was $38.2 \%(102 / 267)$, compared to $14.8 \%(78 / 526)$ among adults aged 52-54 years $(\mathrm{OR}=3.21$; $95 \% \mathrm{CI}$ : 2.26-4.57). Other sociodemographic predictors of health literacy decline were: male sex $(\mathrm{OR}=1.20$; $95 \% \mathrm{CI}$ : 1.04 1.38), non-white ethnicity (OR=2.42; 95\% CI: 1.51-3.89), low educational attainment $(\mathrm{OR}=1.58$; $95 \% \mathrm{CI}$ : 1.29 1.95 for no qualifications vs. degree education), and low occupational class $(\mathrm{OR}=1.67$; $95 \% \mathrm{CI}$ : 1.39-2.01 for routine vs. managerial occupations). Higher baseline cognitive function scores protected against health literacy decline, while cognitive decline (yes vs. no) predicted decline in health literacy score $(\mathrm{OR}=1.59 ; 95 \% \mathrm{CI}$ : 1.35-1.87 for memory decline and $\mathrm{OR}=1.56$; $95 \% \mathrm{CI}: 1.32-1.85$ for executive function decline).

CONCLUSIONS: Health literacy decline appeared to increase with age, and was associated with even subtle cognitive decline in older non-impaired adults. Striking social inequalities were evident, whereby men and those from minority and deprived backgrounds were particularly vulnerable to literacy decline. Health practitioners must be able to recognize limited health literacy to ensure that clinical demands match the literacy skills of diverse patients.

Electronic supplementary material The online version of this article (doi:10.1007/s11606-015-3206-9) contains supplementary material, which is available to authorized users.

Received October 18, 2014

Revised December 23, 2014

Accepted January 20, 2015

Published online February 14, 2015
KEY WORDS: health literacy; cognition; aging; epidemiology.

J Gen Intern Med 30(7):958-64

DOI: $10.1007 / \mathrm{s} 11606-015-3206-9$

(C) The Author(s) 2015. This article is published with open access at Springerlink.com

\section{INTRODUCTION}

In North America, over half of all adults and over $70 \%$ of adults aged over 65 years have low health literacy, defined as having trouble accessing, understanding, and using information to make basic health decisions. ${ }^{1,2}$ Low health literacy is associated with taking of prescription medications improperly, excess use of emergency care, less use of preventive care services, and increased risks for morbidity and mortality. ${ }^{3-7}$ Evidently, there is a broad mismatch between individuals' literacy skills and the health management demands placed upon them by health systems, resulting in literacy-based barriers to good health. The improvement of health literacy of populations is a major goal of health organizations including the U.S. Centers for Disease Control and Prevention and the World Health Organization.,

The health consequences of low literacy may be especially pertinent in older populations, given that older adults commonly need health information and services to manage their increasingly complicated health issues. ${ }^{10}$ Cross-sectional research has consistently associated older age with poorer performance on health literacy tests. ${ }^{11}$ Subsequently, health literacy skills are assumed to decline during aging. An important consideration for examining the dynamics of health literacy decline in older populations is cognitive aging, as cognitive function is related to health literacy. Fluid cognitive abilities (e.g., working memory, reasoning) and crystallized cognitive abilities (e.g., vocabulary, generalized knowledge) have been shown to jointly explain over $70 \%$ of the association between health literacy and performance on health-related tasks among older adults. ${ }^{12}$

However, the effect of typical cognitive aging processes on health literacy skills remains unclear. ${ }^{13-19}$ Furthermore, the distribution of health literacy skill decline in an older population has never been demonstrated, an awareness of which would be imperative for researchers, health practitioners, and policymakers, because low health literacy is a major determinant of morbidity and mortality in the United States, England, and globally. 5,6,20 
In this study, we aimed to describe health literacy decline during aging and the potential contributing roles of cognitive function and decline to health literacy decline among noncognitively impaired English adults aged $\geq 52$ years.

\section{METHODS}

\section{The English Longitudinal Study of Ageing}

The English Longitudinal Study of Ageing (ELSA) is a population-based longitudinal cohort study that aims to characterize the economic, social, and health consequences of aging among English adults aged $\geq 50$ years. ${ }^{21}$ The original ELSA cohort of 12,100 adults (response rate $=66 \%$ ) was established in 2002 based on a random stratified sample of households. ${ }^{21}$ ELSA data are collected biennially through computer-assisted interviews. The ELSA was approved by the London Multicentre Research Ethics Committee (MREC/01/2/91) and informed consent was obtained from all participants.

\section{Study Sample}

All ELSA participants from the original cohort who were in the study at waves 2 (2004-2005) and 5 (2010-2011) of data collection were eligible for inclusion. Wave 2 included 8,780 participants of the original 12,100 . Of these, 5,840 were retained at wave 5 (33.5\% attrition between waves 2 and 5$)$ and were eligible for inclusion in the present analysis. Of the 8,780 participants at wave 2, 8,316 (94.7\%) completed the health literacy assessment. Common non-completion reasons were sight difficulties $(n=132)$, health problems $(n=59)$, or that the interview was done by proxy due to physical or cognitive impairment of the participant, and therefore was not eligible for the health literacy assessment $(\mathrm{n}=92)$.

Of the 5,840 core participants in the study at wave 5, 5,330 $(91.3 \%)$ completed the health literacy assessment. Common reasons for non-completion of the health literacy assessment at wave 5 were sight problems $(n=96)$, health problems $(n=37)$, and having a study interview done by proxy $(\mathrm{n}=214)$. In total, $5,256 / 5,840$ participants had data on health literacy at both time points $(90.0 \%)$. Of these, one participant was missing data on education, two on ethnicity, and four on occupational class. Cognitive function data were missing for 257 of these participants $(4.9 \%)$. The univariate analysis of health literacy decline included all 5,256 participants, the multivariable modelling with sociodemographics included 5,252 participants, and the models including cognitive variables included 4,999 participants.

\section{Health Literacy}

Health literacy was assessed using a four-item measure from the Adult Literacy and Life Skills Survey developed by the Organization for Economic Co-operation and Development (OECD) and Statistics Canada. ${ }^{22}$ Participants were required to read a fictitious medicine label similar to that found on an aspirin packet, and were asked four reading comprehension questions about the label by the interviewer (Appendix 1). Adequate health literacy was defined as scoring $4 / 4$ correct on the measure and limited health literacy as scoring $<4 / 4$. Health literacy decline was defined as a decrease of $\geq 1$ point in score between waves.

\section{Cognitive Variables}

Waves 2 and 5 of ELSA collection included an intervieweradministered cognitive battery, which assessed several cognitive processes essential to daily functioning that were sensitive to decline with aging and were measured in a way to prevent ceiling or floor effects. ${ }^{23}$ The cognitive processes assessed were: time orientation (ability to state the correct day, week, month, and year), verbal learning (of ten words presented aurally), immediate and delayed recall (of the same ten words), prospective memory (remembering to write initials on a clipboard at a certain point during the battery after being instructed to do so earlier on), verbal fluency and mental flexibility (the number of animals named in one minute), and a test of attention, visual search, and mental processing speed (the number of target letters in a grid of random alphabet letters crossed out in one minute). ${ }^{23}$ The former four tests were grouped to create an index of memory function, with potential scores ranging from 0 to 27 , and the latter two tests were grouped to create an index of executive function, with potential scores ranging from 0 to no defined upper limit. ${ }^{23}$ For each index, cognitive decline was defined as a decline of $>1$ point. ${ }^{23}$ Memory and executive function collectively will be referred to as 'cognitive function' and collective decline as 'cognitive decline' throughout this paper.

\section{Sociodemographic Covariates}

Sociodemographic covariates obtained from the wave 2 interview were: age in years $(52-54 ; 55-59 ; 60-64 ; 65-69 ; 70-74$; $75-79 ; \geq 80$ ), sex (male; female), ethnicity (white; non-white), educational attainment (degree or equivalent; up to degree level; no qualification), and occupational class according to the three-category UK National Statistics Socioeconomic Classification (managerial; intermediate; routine). Age began at 52 rather than 50 years because this analysis begins 2 years into ELSA data collection. Education was included as a measure of literacy skills gained through schooling, and occupation as a measure of social standing and of literacy skills used throughout working life.

\section{Statistical Analysis}

The prevalence of limited health literacy was calculated overall and by 5 -year age group at baseline. Mean health literacy scores at each wave were calculated and graphed by 5-year age group, and compared across age groups using the KruskalWallis test and within age groups using the Wilcoxon signrank test for matched pairs. Logistic regression models 
adjusted for all a priori-selected sociodemographic variables were used to estimate odds ratios (ORs) and associated $95 \%$ confidence intervals (CIs) for the associations between age, sociodemographics, and health literacy decline. To prevent baseline adjustment bias, ${ }^{24,25}$ baseline health literacy was not adjusted for in regression modelling, as health literacy decline and score at baseline are both strongly correlated with age and likely share other common causes.

Baseline memory and baseline executive function were added to the model to determine their independent associations with health literacy decline and mediating effects on the association with age; memory decline and executive function decline were then added in a second step. A sensitivity analysis was performed, redefining cognitive decline variables according to increasingly conservative definitions of decline: decreases of $>2$ and $>5$ points on each index. A post-hoc analysis of chronic disease diagnoses that may affect cognition was run to assess their potential additional contribution to explaining the association between age and health literacy. Chronic diseases were diabetes, heart disease (angina, heart attack, abnormal heart rhythm or congestive heart failure), chronic lung disease (chronic bronchitis or emphysema), and depressive symptoms.

The impact of missing data was investigated by running multiple imputations of missing values for health literacy and cognitive function (see full Methods in Appendix 2). All analyses were conducted using StataSE 13.1 (StataCorp, College Station, TX).

\section{RESULTS}

At baseline, 1,455/5,256 (27.7 \%) participants had limited health literacy (Table 1). When followed forward to wave 5 , $3,260 / 5,256$ participants $(62.0 \%)$ had no change in their health literacy score, while 964/5,256 (18.3\%) improved by $\geq 1$ point and 1,032/5,256 (19.6\%) declined by $\geq 1$ point. Chisquared tests showed that improvement in score was nondifferential by age $(p=0.53)$, while decline was more frequent in older age groups $(p<0.001)$. The proportion that declined increased linearly with age from $14.8 \%(78 / 526)$ of those aged $52-54$ years $(102 / 267)$ to $38.2 \%$ of those aged $\geq 80$ years $(p<0.0001)$. As shown in Fig. 1, mean health literacy scores declined over the study follow-up for age groups from 65 69 years and older; this decline was statistically significant for the 75-79 $(p=0.008)$ and $\geq 80(p<0.001)$ groups.

In the multivariable logistic regression model adjusted for sex, ethnicity, occupation, and education, the OR for health literacy decline among those aged $65-69$ years vs. 52-54 years was 1.34 (95\% CI: 1.00-1.79; Table 2). ORs increased in a linear fashion across age groups $\left(p_{\text {trend }}<0.001\right)$ up to 3.21 (95 \% CI: $2.26-4.57$ ) for the $\geq 80$ vs. the $52-54$ age group. Independent of baseline age, the sociodemographic risk factors for health literacy decline were: being male $(\mathrm{OR}=1.20$; $95 \%$ CI: $1.04-1.38)$, of a non-white ethnicity $(\mathrm{OR}=2.42$;
Table 1. Baseline Characteristics of Study Participants, the English Longitudinal Study of Ageing, 2004-2005 $(n=5,256)$

\begin{tabular}{|c|c|}
\hline & N (\%) \\
\hline \multicolumn{2}{|l|}{ Age } \\
\hline $52-54$ & $526(10 \%)$ \\
\hline $55-59$ & $1,346(26 \%)$ \\
\hline $60-64$ & $995(19 \%)$ \\
\hline $65-69$ & $952(18 \%)$ \\
\hline $70-74$ & $692(13 \%)$ \\
\hline $75-79$ & $478(9 \%)$ \\
\hline$\geq 80$ & $267(5 \%)$ \\
\hline \multicolumn{2}{|l|}{ Health literacy } \\
\hline Limited & $1,455(28 \%)$ \\
\hline \multicolumn{2}{|l|}{ Sex } \\
\hline Female & $2,960(56 \%)$ \\
\hline \multicolumn{2}{|l|}{ Ethnicity } \\
\hline Non-white & $85(2 \%)$ \\
\hline \multicolumn{2}{|l|}{ Occupational class } \\
\hline Managerial & $1,797(34 \%)$ \\
\hline Intermediate & $1,364(26 \%)$ \\
\hline Routine & $2,040(39 \%)$ \\
\hline Other & $53(1 \%)$ \\
\hline \multicolumn{2}{|l|}{ Educational attainment } \\
\hline Degree or equivalent & $1,268(24 \%)$ \\
\hline Up to degree level & $2,349(45 \%)$ \\
\hline No qualification & $1,635(31 \%)$ \\
\hline \multicolumn{2}{|l|}{ Memory index $(/ 27)$} \\
\hline Mean (SD) & $16.57(3.74)$ \\
\hline Median & 17 \\
\hline Range & $4-27$ \\
\hline \multicolumn{2}{|l|}{ Executive function index } \\
\hline Mean (SD) & $13.66(3.10)$ \\
\hline Median & 14 \\
\hline Range & $4-23$ \\
\hline
\end{tabular}

$95 \%$ CI: 1.51-3.89), being in an occupational class lower than professional $/$ managerial $(\mathrm{OR}=1.35 ; 95 \% \mathrm{CI}: 1.11-1.65$ for intermediate; $\mathrm{OR}=1.67$; $95 \% \mathrm{CI}$ : $1.39-2.01$ for routine; $\mathrm{OR}=1.90 ; 95 \% \mathrm{CI}: 1.02-3.53$ for 'other'), and having no educational qualifications $(\mathrm{OR}=1.58 ; 1.29-1.95)$.

Mean baseline memory and executive function decreased with age $(p<0.0001)$. Higher baseline cognitive function was protective against health literacy decline regardless of age, where every 1-point increase in memory score was associated with an OR of 0.94 (95\% CI: 0.91-0.96) and every 1-point increase in executive function score was associated with an OR of 0.92 (95 \% CI: 0.89-0.94) for health literacy decline (Table 2). The likelihood of cognitive decline over the followup period increased with age: $30.9 \%(162 / 525)$ of those aged 52-54 experienced memory decline, compared with $55.2 \%$ $(144 / 261)$ of those aged $\geq 80$; the corresponding values for executive function decline were $25.6 \%(128 / 500)$ and $45.3 \%$ $(111 / 245)(p<0.001$ for both). As shown in Table 2, memory and executive function decline were associated with health literacy decline independent of age (ORs $=1.59 ; 95 \%$ CI: $1.35-1.87$ and 1.56 ; $95 \%$ CI: 1.32-1.85). Baseline cognitive function and cognitive decline over the follow-up period explained most of the association between health literacy decline and age. The associations between other sociodemographic variables and health literacy decline persisted regardless of adjustment for cognition (Table 2).

When memory decline was defined as declines of $>2$ and $>$ 5 points, 1,386/4,999 (27.7\%) and 485/4,999 (9.7\%) 


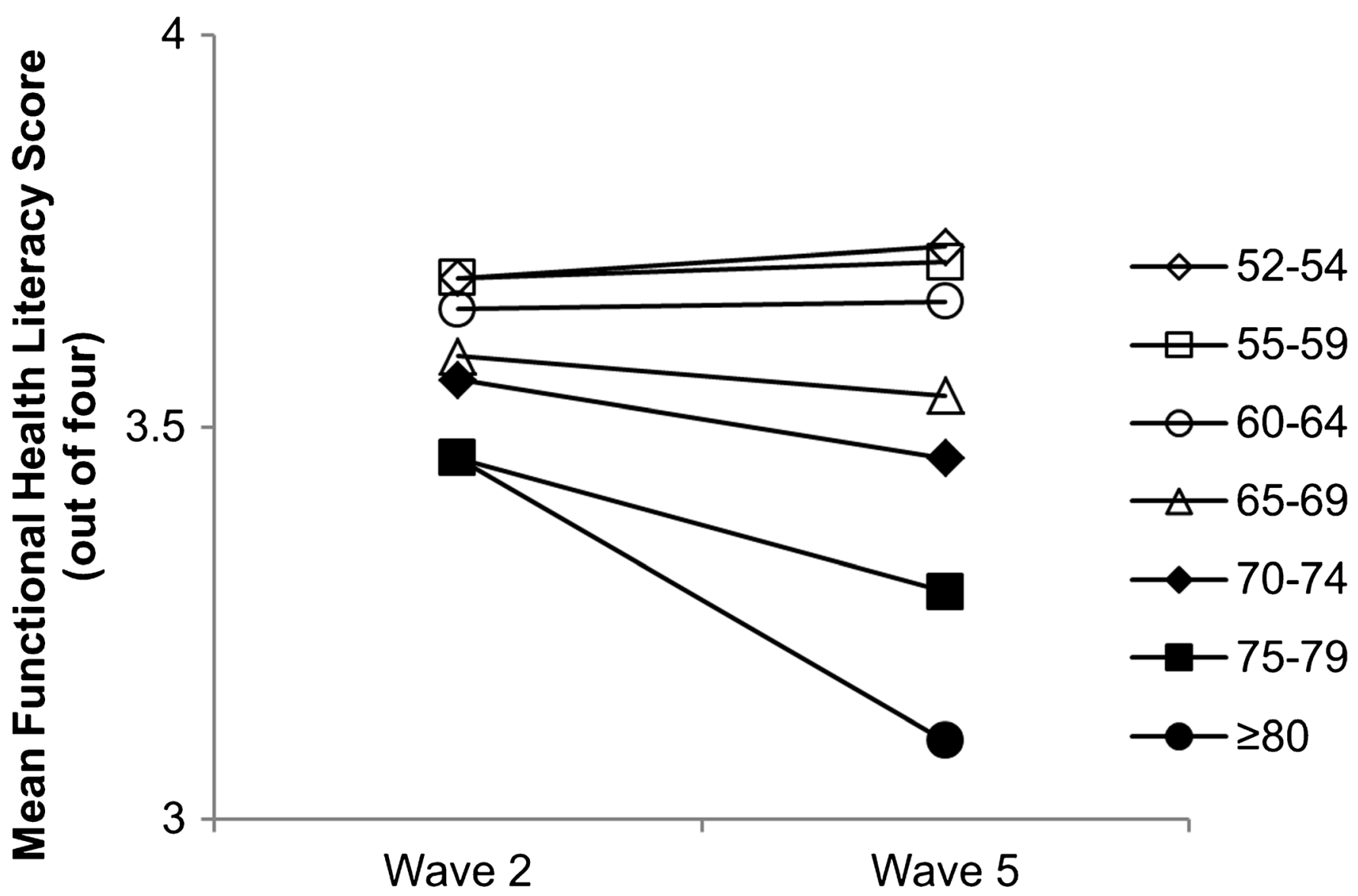

ELSA Wave

Figure 1. Mean health literacy scores between ELSA waves 2 (2004-2005) and 5 (2010-2011) by 5-year age group.

participants were defined as experiencing memory decline. The corresponding values for these re-definitions of executive function decline were 988/4,999 (19.8\%) and 138/4,999 $(2.8 \%)$. When each of these increasingly conservative definitions was used to create the cognitive decline variables, results were unaltered from the original analysis. Having a chronic disease diagnosis or depressive symptoms was not associated with health literacy decline when added to the final model. Finally, results from the multiple imputation analysis were mostly similar to the complete-case analysis (Appendix 2).

\section{DISCUSSION}

Nearly one-third of English adults aged 52 years and over had health literacy limitations in this large longitudinal study. Over the 6-year follow-up period, one-fifth of the sample declined in health literacy skills. Age differences in the likelihood and rate of health literacy decline were pronounced, with adults over age 80 having over three times greater odds of experiencing health literacy decline than those in their early 50s. Striking social inequalities in health literacy decline were evident, where men, ethnic minorities, those with no educational qualifications, and those with a lower occupational class were vulnerable to loss of the literacy skills required to manage health during aging. Cognition appears to be a key risk factor explaining health literacy decline. Even subtle, onepoint differences in cognitive function affected the likelihood of health literacy decline, and experiencing cognitive decline of any magnitude was strongly associated with health literacy decline.

This study is the first and the largest to our knowledge to track health literacy skills over time, particularly among an aging sample. Our finding that cognitive function mostly explained the relationship between older age and health literacy decline was expected, based on cross-sectional evidence showing that the constructs of cognition and health literacy overlap to a large degree. ${ }^{12,13,18,26}$ Contrary to our findings, the association between age and health literacy was independent of cognitive impairment according to the Mini Mental Status Examination (MMSE) score in previous research, ${ }^{14,16,17}$ although the MMSE does not detect subtle individual differences in cognitive function. An important aspect of our study is that not everyone who experienced cognitive decline also experienced health literacy decline. The degree to which typical cognitive aging versus aging- 
Table 2. Multivariable-Adjusted Associations Between Age, Sociodemographic Factors, Cognition, and Health Literacy Decline, the English Longitudinal Study of Ageing, England, 2004-2011 ( $\mathrm{n}=5256)$

\begin{tabular}{|c|c|c|c|}
\hline & \multicolumn{3}{|c|}{ Odds ratios for health literacy decline } \\
\hline & $\mathrm{OR}^{*}(95 \% \mathrm{CI})$ & $\mathrm{OR}^{\dagger}(95 \% \mathrm{CI})$ & $\mathrm{OR}^{\star}(95 \% \mathrm{CI})$ \\
\hline \multicolumn{4}{|l|}{ Age } \\
\hline $52-54$ & 1.00 (Reference) & 1.00 (Reference) & 1.00 (Reference) \\
\hline $55-59$ & $0.92(0.69-1.23)$ & $0.95(0.70-1.28)$ & $0.93(0.68-1.26)$ \\
\hline $60-64$ & $1.07(0.80-1.44)$ & $1.04(0.76-1.41)$ & $0.98(0.72-1.34)$ \\
\hline $65-69$ & $1.34(1.00-1.79)$ & $1.21(0.90-1.65)$ & $1.08(0.79-1.48)$ \\
\hline $70-75$ & $1.53(1.13-2.07)$ & $1.26(0.91-1.74)$ & $1.07(0.77-1.48)$ \\
\hline $75-79$ & $1.94(1.41-2.67)$ & $1.43(1.01-2.03)$ & $1.09(0.76-1.55)$ \\
\hline$\geq 80$ & $3.21(2.26-4.57)$ & $2.26(1.54-3.32)$ & $1.65(1.11-2.45)$ \\
\hline \multicolumn{4}{|c|}{ (2.24 (2.20 } \\
\hline Male & $1.20(1.04-1.38)$ & $1.10(0.94-1.27)$ & $1.05(0.90-1.22)$ \\
\hline \multicolumn{4}{|l|}{ Ethnicity } \\
\hline Non-white & $2.42(1.51-3.89)$ & $1.81(1.10-2.99)$ & $1.67(1.01-2.77)$ \\
\hline \multicolumn{4}{|l|}{ Occupational class } \\
\hline Managerial & 1.00 (Reference) & 1.00 (Reference) & 1.00 (Reference) \\
\hline Intermediate & $1.35(1.11-1.65)$ & $1.26(1.02-1.55)$ & $1.24(1.01-1.53)$ \\
\hline Routine & $1.67(1.39-2.01)$ & $1.50(1.23-1.82)$ & $1.43(1.18-1.74)$ \\
\hline Other & $1.90(1.02-3.53)$ & $1.60(0.83-3.06)$ & $1.44(0.75-2.77)$ \\
\hline \multicolumn{4}{|l|}{ Educational attainment } \\
\hline Degree or equivalent & 1.00 (Reference) & 1.00 (Reference) & 1.00 (Reference) \\
\hline Up to degree level & $1.04(0.86-1.26)$ & $1.06(0.87-1.30)$ & $1.07(0.88-1.31)$ \\
\hline No qualification & $1.58(1.29-1.95)$ & $1.33(1.07-1.66)$ & $1.30(1.04-1.62)$ \\
\hline \multicolumn{4}{|l|}{ Baseline memory } \\
\hline Per 1-point score increase & - & $0.96(0.93-0.98)$ & $0.94(0.91-0.96)$ \\
\hline \multicolumn{4}{|l|}{ Baseline executive function } \\
\hline Per 1-point score increase & - & $0.93(0.91-0.96)$ & $0.92(0.89-0.94)$ \\
\hline \multicolumn{4}{|l|}{ Memory decline } \\
\hline Yes & - & - & $1.59(1.35-1.87)$ \\
\hline \multicolumn{4}{|l|}{ Executive function decline } \\
\hline Yes & - & - & $1.56(1.32-1.85)$ \\
\hline
\end{tabular}

*Adjusted for covariates (age, sex, ethnicity, occupational class, educational attainment)

${ }^{*}$ Adjusted for covariates, and baseline memory \& executive function; $n=4999$

${ }^{*}$ Adjusted for covariates, baseline memory \& executive function, and memory \& executive function decline; $n=4999$

related cognitive impairments of varying severities affect health literacy skills remains to be elucidated. Our study suggests that non-pathological cognitive decline negatively affects health literacy during aging. Further longitudinal studies that address the fluidity of literacy and cognition during aging are needed for consideration alongside ours.

We observed a degree of health literacy decline among adults aged $\geq 80$ years not explained by cognition, which may be because we could not account for all aspects of cognitive function. For example, inductive reasoning was not measured, but is correlated with both age and health literacy. ${ }^{12,27}$ Visual and auditory functioning also play roles in one's ability to take in and learn from new information. We did not account for these factors, although participants unable to take the test due to sensory limitations were excluded. We also had no measures of the component processes involved with active learning, including knowledge integration and text inference, which predict reading comprehension skills among older adults. ${ }^{28}$ However, the aspects of short-term memory and processing speed that we measured are related to these abilities. It may also be that other factors besides cognitive function influence potential generational differences in likelihood of literacy skill loss, such as lifetime educational experiences.

Although validation data for the individual health literacy measure we used were not available, the measure was taken from a validated international adult literacy survey. ${ }^{22}$ The measure does not capture prose literacy, information navigation, or numeracy, although it is a measure of document literacy that has good face validity. The ability to read and understand a medicine label is crucial to several health outcomes, and has been associated with risk of all-cause mortality among older adults. ${ }^{5}$ The scale had narrow range and a ceiling effect, where over two-thirds of our study sample scored $4 / 4$ on the scale at both time points; this is a common problem in health literacy measures. ${ }^{29,30}$ Consequently, few participants declined by $>1$ point (only 316/5,256; $6 \%$ ), preventing us from examining decline of varying magnitudes and from varying starting points. We could not examine non-linear change or change longer than our 6-year follow-up period. However, as a longitudinal analysis conducted with little prior knowledge on health literacy during aging, this study provides valuable evidence for future research hypotheses.

Another important limitation of this study is attrition bias. The prevalence of limited health literacy at baseline was $42 \%$ among those who dropped out of the study, but was only $28 \%$ among those who remained in the study between waves. Study attrition also increased with age, from approximately $26 \%$ among those aged 52-54 years to $71 \%$ among those aged $\geq$ 80 years. Our results may therefore underestimate the true prevalence of limited health literacy among the older English population, particularly in the most elderly age group. Ethnic minorities, participants with no educational qualifications, and 
those with routine occupations were more also likely to drop out of the study, and were more likely to have limited health literacy at baseline. Therefore, we may have underestimated the magnitude of associations between these sociodemographic variables and health literacy decline. Reassuringly, missing data do not seem to introduce notable bias into our results, as results from the multiple imputation analysis were similar to those from the complete-case analysis.

Future work should investigate more comprehensive aspects of cognition including reasoning and sensory functioning to elucidate the role of cognition in health literacy decline. Longitudinal data should be collected at multiple time points to examine non-linear trajectories of health literacy change over time and for longer follow-up periods than 6 years. Potentially modifiable behavioral and health-related influences on health literacy are unknown. For example, internet use and engagement in regular reading may help adults to maintain health literacy during aging through directly stimulating cognitive and literacy skills. Diagnoses of health conditions, physical functioning, and experiences with the health care system may affect health literacy in multiple complex ways. Future research should investigate these and other potential influences on health literacy decline; if certain practices can help maintain health literacy skills regardless of cognitive aging, this evidence will inform the development of interventions to improve literacy skills in health settings.

\section{CONCLUSIONS}

The literacy skills required to manage health appear to undergo aging-related decline among older English adults beginning around age 65 . Rate of decline increases with age, with adults aged $\geq 80$ years being vulnerable to rapid health literacy decline. Health literacy among older adults is marked by social inequalities, whereby men and adults from deprived social groups are the most vulnerable to skill loss during aging. Cognitive function and even slight cognitive decline during aging appear to affect the likelihood of health literacy decline. Finally, given that literacy skills are commonly lost during aging, a time when adults often need health information and services, the current population-wide burden of low health literacy may be substantial. Innovative interventions to help reduce and prevent literacy barriers to good health during aging are needed, along with individual support from practitioners in daily practice.

Contributors: We thank Dr Aparna Shankar, Dr Sophie Bostock, and Prof Andrew Steptoe for their assistance with data acquisition.

Funding: The English Longitudinal Study of Ageing is funded by the National Institute of Aging in the United States (grant numbers 2RO1AG7644-01A1, 2RO1AG017644), and a consortium of UK government departments coordinated by the Office for National Statistics. The present analysis was supported by a Doctoral Foreign Study Award (DFSA 201210) from the Canadian Institutes of Health Research and an Overseas Research Scholarship from University College London to Lindsay C Kobayashi, and a program grant from Cancer Research UK
(C1418/A14134) to Jane Wardle. The funders had no role in the design or conduct of the present study; collection, management, analysis, and interpretation of data; or preparation, review, or approval of the manuscript; and decision to submit the manuscript for publication.

Prior Presentations: Earlier versions of this work were presented orally at the Ninth Annual Scientific Meeting of the UK Society for Behavioural Medicine in Oxford, UK on 10 December 2013 and the Second European Health Literacy Conference in Aarhus, Denmark on 11 April 2014.

Conflict of Interest: Michael S Wolf has consulted for Vivus, Abbott, Abbvie, Merck, UnitedHealthcare, and Luto and has received research grants from Abbott, Abbvie, Merck and UnitedHealthcare within the past 3 years, and has been a continuing medical education speaker for MedLearning Group. Lindsay C Kobayashi, Jane Wardle, and Christian von Wagner have no potential conflicts of interest to declare.

Corresponding Author: Lindsay C. Kobayashi, MSc; Health Behaviour Research Centre, Department of Epidemiology and Public HealthUniversity College London, 1-19 Torrington Place, 2nd floor, London, UK WC1E 6BT (e-mail: l.kobayashi.12@ucl.ac.uk).

Open Access This article is distributed under the terms of the Creative Commons Attribution License which permits any use, distribution, and reproduction in any medium, provided the original author(s) and the source are credited.

\section{REFERENCES}

1. Institute of Medicine. In: Nielsen-Bohlman L, Panzer A, Hamlin B, Kindig D, eds. Health literacy: a prescription to end confusion. Washington D.C.: National Academies Press; 2004.

2. Kutner M, Greenberg E, Jin Y, Paulsen C. The Health Literacy of America's adults: results from the 2003 National Assessment of Adult Literacy (NCES 2006-483). Washington D.C.; 2006 p. 60.

3. von Wagner C, Knight $\mathbf{K}$, Steptoe A, Wardle J. Functional health literacy and health-promoting behaviour in a national sample of British adults. $J$ Epidemiol Community Health. 2007;61(12):1086-90.

4. Berkman ND, Sheridan SL, Donahue KE, Halpern DJ, Crotty K. Low Health Literacy and Health Outcomes: An Updated Systematic Review. Ann Intern Med. 2011;155:97-107.

5. Bostock S, Steptoe A. Association between low functional health literacy and mortality in older adults: longitudinal cohort study. BMJ. 2012;344:e1602.

6. Baker DW, Wolf MS, Feinglass J, Thompson JA, Gazmararian JA, Huang $\mathbf{J}$. Health literacy and mortality among elderly persons. Ann Intern Med. 2007;167(14):1503-9.

7. Kobayashi LC, Wardle J, von Wagner C. Limited health literacy is a barrier to colorectal cancer screening in England: Evidence from the English Longitudinal Study of Ageing. Prev Med. 2014;61:100-5.

8. World Health Organization. Track 2: Health literacy and health behaviour. 7th Global Conference on Health Promotion: track themes. Available at: http://www.who.int/healthpromotion/conferences/7gchp/track2/en/. Accessed October 7, 2014.

9. U.S. Centers for Disease Control and Prevention. Health Literacy. Available at: http://www.cdc.gov/healthliteracy/introduction.html. Accessed October 7, 2014.

10. Parker R, Ratzan S, Lurie N. Health Literacy: A policy challenge for advancing high-quality health care. Health Aff (Millwood). 2003;22(4):147-53.

11. Kobayashi LC, Wardle J, Wolf MS, von Wagner C. Aging and functional health literacy: a systematic review and meta-analysis. J Gerontol B Psychol Sci Soc Sci. 2014. In press.

12. Wolf MS, Curtis LM, Wilson EAH, et al. Literacy, cognitive function, and health: results of the LitCog study. J Gen Intern Med. 2012;27(10):1300-7.

13. Kaphingst KA, Goodman MS, Macmillan WD, Carpenter CR, Griffey RT. Effect of cognitive dysfunction on the relationship between age and health literacy. Patient Educ Couns. 2014;95(2):218-25.

14. Armistead-Jehle P, Cifu DX, Wetzel R, Carne W, Klanchar LA. Health literacy among patients diagnosed with movement disorders: a pilot study. PMR. 2010;2(1):43-7.

15. Chew LD, Bradley KA, Flum DR, Cornia PB, Koepsell TD. The impact of low health literacy on surgical practice. Am J Surg. 2004; 188:250-3. 
16. Gazmararian JA, Baker DW, Williams MV, et al. Health literacy among Medicare enrollees in a managed care organization. JAMA. 1999;281(6):545-51.

17. Baker D, Gazmararian J, Sudano J, Patterson M, Parker R, Williams M. Health literacy and performance on the Mini-Mental State Examination. Aging Ment Health. 2002;6(1):22-9.

18. Levinthal BR, Morrow DG, Tu W, Wu J, Murray MD. Cognition and health literacy in patients with hypertension. $J$ Gen Intern Med. 2008;23(8):1172-6.

19. Morrow D, Clark D, Tu W, et al. Correlates of health literacy in patients with chronic heart failure. Gerontologist. 2006;46(5):669-76.

20. Kickbusch I, Pelikan JM, Apfel F, Tsouros AD. Health literacy: The solid facts. Copenhagen; 2013 p. 86.

21. NatCen Social Research. English Longitudinal Study of Ageing (ELSA) Wave One to Wave 5: User Guide to the datasets. Available at: http://www.elsaproject.ac.uk/publicationDetails/id/6791. Accessed December 17, 2014.

22. Thorn W. International Adult Literacy and Basic Skills Surveys in the OECD Region. 2009. Report No.: 26.

23. Banks J, Breeze E, Cheshire $\mathbf{H}$, et al. Cognitive function. In: Banks J, Breeze E, Lessof C, Nazroo J, eds. Retirement, health and relationships of the older population in England: The 2004 English Longitudinal Study of Ageing (Wave 2). London: The Institute for Fiscal Studies; 2006:217-42.
24. Dugravot A, Guéguen A, Kivimaki M, et al. Socioeconomic position and cognitive decline using data from two waves: what is the role of the wave 1 cognitive measure? J Epidemiol Community Health. 2009;63(8):675-80.

25. Glymour MM, Weuve J, Berkman LF, Kawachi I, Robins JM. When is baseline adjustment useful in analyses of change? An example with education and cognitive change. Am J Epidemiol. 2005;162(3): 267-78.

26. Mõttus R, Johnson W, Murray C, Wolf MS, Starr JM, Deary IJ. Towards understanding the links between health literacy and physical health. Health Psychol. 2014;33(2):164-73.

27. Singh-Manoux A, Kivimaki M, Glymour M, et al. Timing of onset of cognitive decline: results from Whitehall II prospective cohort study. BMJ. 2012;344:d7622.

28. Hannon B, Daneman M. Age-related changes in reading comprehension: an individual-differences perspective. Exp Aging Res. 2009;35(4):432-56.

29. Parker R, Baker D, Williams M, Nurss J. The test of functional health literacy in adults: a new instrument for measuring patients' literacy skills. J Gen Intern Med. 1995;10(10):537-41.

30. Davis T, Long S, Jackson R, Mayeaux E, George R, Murphy P, et al. Rapid assessment of adult literacy in medicine: a shortened screening instrument. Fam Med. 1993;25(6):391-5. 